\title{
Counting topologies
}

Gerald Kuba

Gerald Kuba promovierte 1991 an der Universität Wien und ist seit 2001 Dozent für Mathematik ebendort, sowie außerordentlicher Professor an der Universität für Bodenkultur Wien. Sein primäres Forschungsgebiet ist die Gitterpunktlehre, daneben gilt sein Interesse grundlegenden Problemen aus klassischer Analysis und Algebra.

\section{Statement of results}

Write $|M|$ for the cardinal number of a set $M$ and let $\mathcal{P}(M)$ denote the power set of $M$, $\mathcal{P}(M)=\{X \mid X \subset M\}$. If $|M|=\kappa$ then $|\mathcal{P}(M)|=2^{\kappa}$. In particular, $c=2^{\aleph_{0}}$ where as usual $\aleph_{0}=|\mathbb{N}|$ and $c=|\mathbb{R}|$. For any transfinite cardinal $\kappa$ let $\kappa^{+}$denote the least cardinal number greater than $\kappa$. (For example, $\aleph_{0}^{+}=\aleph_{1}, \aleph_{1}^{+}=\aleph_{2}, \ldots$ ) Naturally, $\kappa<\kappa^{+} \leq 2^{\kappa}$. Neither $\kappa^{+}=2^{\kappa}$ nor $\kappa^{+}<2^{\kappa}$ is provable for any transfinite cardinal $\kappa$.

Fix an infinite set $X$ with $|X|=\kappa$ and let $\mathcal{T}$ denote the family of all topologies $\tau$ on $X$. So we have $\tau \in \mathcal{T}$ whenever $\tau \subset \mathcal{P}(X)$ and $X$ becomes a topological space by declaring a set $U \subset X$ open if and only if $U \in \tau$. Let us call two topologies $\tau_{1}, \tau_{2}$ isomorphic when the two spaces $\left(X, \tau_{1}\right)$ and $\left(X, \tau_{2}\right)$ are homeomorphic. Clearly, being isomorphic defines an equivalence relation on every family $\mathcal{F} \subset \mathcal{T}$. Let $\mathcal{F}^{*}$ be the quotient set of $\mathcal{F}$ with respect to this equivalence relation. We are interested in computing the cardinal numbers $|\mathcal{F}|$ and $\left|\mathcal{F}^{*}\right|$ where $\mathcal{F}$ is the family of all $\tau \in \mathcal{T}$ such that the topological space $(X, \tau)$ has a certain property $(\mathrm{P})$. Hence $|\mathcal{F}|$ is the total number of topologies $\tau$ on $X$ where the space $(X, \tau)$

Mit $\mathcal{P}(M)$ sei die Potenzmenge der Menge $M$ bezeichnet. Auf einer vorgegebenen unendlichen Menge $X$ kann man auf viele Arten Topologien definieren. Genauer ist die Familie $\mathcal{T}$ aller topologischen Räume, denen $X$ als Punktmenge zugrunde liegt, gleichmächtig mit der Menge $\mathcal{P}(\mathcal{P}(X))$. Der Autor zeigt in der vorliegenden Arbeit, dass diese sehr große Mächtigkeit der Familie $\mathcal{T}$ erhalten bleibt, wenn man fordert, dass die Räume in $\mathcal{T}$ beispielsweise alle zusammenhängend oder alle kompakt oder alle Hausdorffsch sein sollen. Auch für gewisse Kombinationen dieser grundlegenden topologischen Eigenschaften trifft dies $\mathrm{zu}$, nicht jedoch für die wichtige Familie aller kompakten Hausdorffräume, welche mit $X$ als Punktmenge nur mit $\mathcal{P}(X)$ gleichmächtig ist. 
satisfies $(\mathrm{P})$ and $\left|\mathcal{F}^{*}\right|$ is the maximal number of mutually non-homeomorphic spaces of size $\kappa$ satisfying $(\mathrm{P})$. In view of $\mathcal{T} \subset \mathcal{P}(\mathcal{P}(X))$ we always have $\left|\mathcal{F}^{*}\right| \leq|\mathcal{F}| \leq 2^{2^{\kappa}}$.

The properties $(\mathrm{P})$ which we are interested in are several combinations of connectedness, compactness and the following separation axioms: T1 (all points are closed), T2 (being Hausdorff), T4 (disjoint closed sets can always be separated by open sets), T5 (every subspace is T4). Please note that we do not assume that compact spaces are automatically Hausdorff and that we also follow [4] in calling a space normal when it satisfies T2 and T4 (or, equivalently, T1 and T4) and completely normal when it satisfies T2 and T5.

Theorem 1. Let $\mathcal{F}_{i}$ be the family of all $\tau \in \mathcal{T}$ such that the space $(X, \tau)$ is compact and connected and $\mathrm{T} 1$ when $i=1$, compact and connected and $\mathrm{T} 4$ when $i=2$, completely normal when $i=3$, connected and Hausdorff when $i=4$. Then $\left|\mathcal{F}_{i}^{*}\right|=\left|\mathcal{F}_{i}\right|=2^{2^{\kappa}}$ for each $i \in\{1,2,3,4\}$. Moreover, $\left|\left(\mathcal{F}_{3} \cap \mathcal{F}_{4}\right)^{*}\right|=2^{2^{\kappa}}$ if $\kappa \geq c$ and $\mathcal{F}_{3} \cap \mathcal{F}_{4}=\emptyset$ if $\kappa<c$.

The next theorem shows that the important combination of compactness and T2 occurs relatively rarely.

Theorem 2. Let $\mathcal{F}_{0}$ be the family of all $\tau \in \mathcal{T}$ such that $(X, \tau)$ is a compact Hausdorff space. Then $\left|\mathcal{F}_{0}\right|=2^{\kappa}$ and $\left|\mathcal{F}_{0}^{*}\right| \geq \kappa^{+}$. But neither $\left|\mathcal{F}_{0}^{*}\right|=\kappa^{+}$nor $\left|\mathcal{F}_{0}^{*}\right|>\kappa^{+}$is provable if $\kappa>\aleph_{0}$.

Since a compact Hausdorff space is second countable if it is countable, the following two theorems imply that the size of $\mathcal{F}_{0}^{*}$ can be determined precisely when either $\kappa \geq c$ or $\kappa=\aleph_{0}$. This does not make the estimate $\left|\mathcal{F}_{0}^{*}\right| \geq \kappa^{+}$superfluous because one cannot rule out that there exist very many cardinals between $\aleph_{0}$ and $c$. (In fact, it is consistent with standard set theory that the set of all cardinals $\lambda$ with $\aleph_{0}<\lambda<c$ has size $c$.)

Theorem 3. If $\kappa<c$ then $\mathcal{F}_{0} \cap \mathcal{F}_{4}=\emptyset$. If $\kappa \geq c$ then $\left|\left(\mathcal{F}_{0} \cap \mathcal{F}_{3} \cap \mathcal{F}_{4}\right)^{*}\right|=2^{\kappa}$.

Theorem 4. Let $\mathcal{F}_{\omega}$ be the family of all $\tau \in \mathcal{T}$ such that $(X, \tau)$ is a second countable (or, equivalently, metrizable) compact Hausdorff space. Then $\mathcal{F}_{\omega}=\emptyset$ when $\kappa>\aleph_{0}$ and $\kappa \neq c$. If $\kappa=\aleph_{0}$ then $\left|\mathcal{F}_{\omega}\right|=c$ and $\left|\mathcal{F}_{\omega}^{*}\right|=\aleph_{1}$. If $\kappa=c$ then $\left|\mathcal{F}_{\omega}\right|=\left|\mathcal{F}_{\omega}^{*}\right|=c$.

\section{Proof of Theorem 1}

The following lemma is useful in order to get rid of isomorphic topologies provided that there are enough topologies at all.

Lemma 1. Let $\mathcal{F} \subset \mathcal{T}$. If $|\mathcal{F}|>2^{\kappa}$ then $\left|\mathcal{F}^{*}\right|=|\mathcal{F}|$.

Proof. Evidently, there are precisely $2^{\kappa}$ permutations on $X$. Hence there are at most $2^{\kappa}$ isomorphic topologies on $X$. Therefore the family $\mathcal{F}^{*}$ is a partition of $\mathcal{F}$ where no equivalence class is larger than $2^{\kappa}$. Thus $\left|\mathcal{F}^{*}\right|<|\mathcal{F}|$ is only possible if $|\mathcal{F}| \leq 2^{\kappa}$.

In view of Lemma 1 it is enough to construct subfamilies $\mathcal{G}_{i} \subset \mathcal{F}_{i}$ with $\left|\mathcal{G}_{i}\right|=2^{2^{\kappa}}$ for $i=1,2,3,4$ in order to prove Theorem 1. It is not surprising that we use ultrafilters in order to achieve the enormous cardinality $2^{2^{\kappa}}$. Let $\mathbf{U}$ be the family of all nonprincipal 
ultrafilters on a fixed set $Y$ with $|Y|=\kappa$, whence $|\mathbf{U}|=2^{2^{\kappa}}$. In the following four examples for each $\mathcal{U} \in \mathbf{U}$ we define topologies $\tau_{1}[\mathcal{U}], \tau_{2}[\mathcal{U}], \tau_{3}[\mathcal{U}], \tau_{4}[\mathcal{U}]$ on $X$ such that for $i=1,2,3,4$ we have $\tau_{i}[\mathcal{U}] \in \mathcal{F}_{i}$ for all $\mathcal{U} \in \mathbf{U}$ and $\tau_{i}[\mathcal{U}] \neq \tau_{i}\left[\mathcal{U}^{\prime}\right]$ whenever $\mathcal{U}, \mathcal{U}^{\prime} \in \mathbf{U}$ are distinct. In Example 1, 2, 3 we identify $X$ with $\{z\} \cup Y$ where $z \notin Y$.

Example 1. For $\mathcal{U} \in \mathbf{U}$ we define the topology $\tau_{1}[\mathcal{U}]$ on $X=\{z\} \cup Y$ in the following way: The empty set and every set $U$ in $\mathcal{U}$ is open. Additionally, a set $\{z\} \cup G$ is open whenever $G \subset Y$ and $Y \backslash G$ is finite. Indeed, $\tau_{1}[\mathcal{U}]$ is a topology since every cofinite set $G \subset Y$ must be an element of the ultrafilter $\mathcal{U}$. It is plain that the space $X$ is T1 and compact. The space $X$ is connected as well because the intersection of two nonempty open sets is never empty since no finite set can be a member of a nonprincipal ultrafilter.

Example 2. For $\mathcal{U} \in \mathbf{U}$ we define the topology $\tau_{2}[\mathcal{U}]$ on $X=\{z\} \cup Y$ in the following way: The empty set and every set $U$ in $\mathcal{U}$ is open. Additionally, the set $X$ is open. Since $X$ is the only open neighborhood of $z$, the space $X$ is compact and connected. The space is T4 vacuously because every nonempty closed set must contain $z$.

Example 3. For $\mathcal{U} \in \mathbf{U}$ we define the topology $\tau_{3}[\mathcal{U}]$ on $X=\{z\} \cup Y$ in the following way: Every subset of $Y$ is open and every set $\{z\} \cup U$ with $U \in \mathcal{U}$ is open. It is easy to check that $\tau_{3}[\mathcal{U}]$ is a topology on $X$. Obviously, each set $M \subset X$ is closed when $z \in M$. (And $M \subset Y$ is closed if and only if $M \notin \mathcal{U}$.) In particular, for each set $S \subset X$ we have $S \subset \bar{S} \subset S \cup\{z\}$. The space $X$ is Hausdorff because if $x, y$ are distinct points in $X$ then in case $z \notin\{x, y\}$ both sets $\{x\}$ and $\{y\}$ are open and in case $x=z$ the set $\{y\}$ is open and $Y \backslash\{y\}$ lies in $\mathcal{U}$ whence the set $\{z\} \cup(Y \backslash\{y\})$ is open. The space $X$ is also T5 which means that two sets $A, B \subset X$ can always be separated by open sets if $\bar{A} \cap B=A \cap \bar{B}=\emptyset$. Let $A, B \subset X$ be sets like that. If $z \notin A \cup B$ then $A, B \subset Y$ and hence $A$ and $B$ are open. Then $A \subset U$ and $B \subset V$ with the two disjoint open sets $U=A$ and $V=B$. Assume $z \in A \cup B$ and say $z \in A$. Then $z \notin \bar{B}$ (since $A$ and $\bar{B}$ are disjoint) and hence $B=\bar{B} \subset Y$. Thus $B$ is closed and open. Then $A \subset U$ and $B \subset V$ with the two disjoint open sets $U=X \backslash B$ and $V=B$.

Example 4. For the present assume $\kappa \geq c$. Let $H$ be a hedgehog formed from the union of $\kappa$ copies of the interval $[0,1]$ (the spines) by identifying the zero points of each interval. ( $H$ is equipped with a canonical metric $d$ defined via $d(x, y)=|x-y|$ when $x, y$ lie on the same spine and $d(x, y)=d(x, 0)+d(y, 0)$ when $x, y$ lie on distinct spines.) Further identify $Y$ with the set of the midpoints of all spines of the hedgehog. Finally identify $X$ with $H \cup\{z\}$ where $z \notin H$. Now for $\mathcal{U} \in \mathbf{U}$ we define the topology $\tau_{4}[\mathcal{U}]$ on $X$ in the following way: A set of points is open if it is either open in $H$ or equals $\{z\} \cup V$ where $V$ is open in $H$ and $V \cap Y$ is an element of the ultrafilter $\mathcal{U}$. In this way $X$ becomes a topological space with $|X|=\kappa$ such that $X$ is Hausdorff and connected. As we will see, the space $X$ is also completely normal and therefore $\left|\mathcal{F}_{3} \cap \mathcal{F}_{4}\right| \geq 2^{2^{\kappa}}$ when $\kappa \geq c$. This implies the last assertion of Theorem 1 in case that $\kappa \geq c$.

In case $\kappa<c$ the cardinality of the spines is inappropriately large and so we cannot use Example 4 in order to verify $\left|\mathcal{F}_{4}^{*}\right|=2^{2^{\kappa}}$. But everything comes right when we modify Example 4 by replacing each spine in $H$ with a copy of any connected and countable 
Hausdorff space. (Use for example the space 61 in [4].) Keeping connectedness and T2 we have to dispense with T4. Moreover, in case $\kappa<c$ we have $\mathcal{F}_{3} \cap \mathcal{F}_{4}=\emptyset$. Actually, if $S$ is a connected and completely regular space then (in view of the Stone-Čech compactification) the space $S$ can be embedded in a cube $[0,1]^{\Lambda}$ with a suitable index set $\Lambda$. Hence the image of $S$ under any canonical projection of $[0,1]^{\Lambda}$ onto $[0,1]$ is a connected subset of $[0,1]$. Consequently, either $|S|=1$ or $|S| \geq c$.

So the proof of Theorem 1 is almost finished. It remains to verify that the space $X$ in Example 4 is T5. For $M \subset H$ let $\bar{M}^{H}$ denote the closure of $M$ in the subspace $H$. Evidently, $\bar{M}^{H}=H \cap \bar{M}$ for all $M \subset H$. Now let $A, B \subset X$ such that $\bar{A} \cap B=$ $A \cap \bar{B}=\emptyset$. We distinguish the two cases $z \in A \cup B$ and $z \notin A \cup B$. If $z \notin A \cup B$ then $\bar{A}^{H} \cap B=A \cap \bar{B}^{H}=\emptyset$. Since the hedgehog $H$ is a metric space, $H$ is completely normal. Therefore we can find disjoint sets $U, V \subset H$ which are open in $H$ and hence open in $X$ such that $A \subset U$ and $B \subset V$. Now assume $z \in A$. Then $z \notin \bar{B}$ and thus the open set $X \backslash \bar{B}$ contains $z$. Therefore we have $F \in \mathcal{U}$ where $F=Y \backslash \bar{B}$. Now let $A_{1}=(A \backslash\{z\}) \cup F \subset H$. Then $\bar{A}_{1}^{H} \cap B=A_{1} \cap \bar{B}^{H}=\emptyset$ since $F$ is closed in $H$ and disjoint from $\bar{B}$. Now choose open and disjoint sets $U, V \subset H$ such that $A_{1} \subset U$ and $B \subset V$. We have $U \cap Y \in \mathcal{U}$ since $U \supset A_{1} \supset F$ and $F \in \mathcal{U}$. Therefore $U \cup\{z\}$ is open in $X$ and contains $A$ and is disjoint from $V$.

\section{Proof of Theorems 2 and 4}

The weight of a topological space $S$ is the least cardinal $\gamma$ such that $S$ has a base $\mathcal{B}$ with $|\mathcal{B}|=\gamma$. It is well-known (cf. [1, Corollary 2.11]) that a compact Hausdorff space $S$ can be embedded in the product space $[0,1]^{\Gamma}$ where $|\Gamma|$ is the weight of $S$. Since the weight of a compact Hausdorff space cannot be greater than the size of the space (cf. [2, 2.1 and 3.11]), any compact Hausdorff space $S$ can be embedded in the cube $[0,1]^{\Lambda}$ with $|\Lambda|=|S|$. Thus for each $\tau \in \mathcal{F}_{0}$ the space $(X, \tau)$ is homeomorphic with a compact subspace of the cube $[0,1]^{\Lambda}$ where $|\Lambda|=|X|=\kappa$. The compact (and connected) cube $[0,1]^{\Lambda}$ contains precisely $2^{\kappa}$ closed sets and hence precisely $2^{\kappa}$ compact subspaces. Consequently, $\left|\mathcal{F}_{0}^{*}\right| \leq$ $2^{\kappa}$ and this implies $\left|\mathcal{F}_{0}\right| \leq 2^{\kappa}$ in view of Lemma 1 . Thus, in order to prove Theorem 2 we have to verify $\left|\mathcal{F}_{0}\right| \geq 2^{\kappa}$ and $\left|\mathcal{F}_{0}^{*}\right| \geq \kappa^{+}$. This is done by the following example.

Example 5. Let $\beta$ be an ordinal number such that there are precisely $\kappa$ limit ordinals below $\beta$. (If $\kappa>\aleph_{0}$ then we can put $\beta=\kappa$ regarding $\kappa$ as an ordinal number. If $\kappa=\aleph_{0}$ then we can put $\beta=\omega^{2}=\sup \{\omega, \omega+\omega, \omega+\omega+\omega, \ldots\}$.) Identify $X$ with the set $[0, \beta]$ of all ordinals $\leq \beta$ and let $\tau$ be the order topology on $X$. Evidently, $(X, \tau)$ is a compact Hausdorff space of size $\kappa$. Let $L$ be the set of all limit ordinals in $X$. For $x \in X$ the singleton $\{x\}$ is open (referring to $\tau$ ) if and only if $x=0$ or $x \notin L$. For

$$
N_{i}:=\{\alpha+i \in X \mid \alpha \in L\} \quad(i \in\{1,2\})
$$

we have $N_{1} \cap N_{2}=\emptyset$ and $L \cap\left(N_{1} \cup N_{2}\right)=\emptyset$. It is clear that

$$
|L|=\left|N_{1}\right|=\left|N_{2}\right|=|X \backslash L|=\left|X \backslash\left(N_{1} \cup N_{2}\right)\right|=|X|=\kappa .
$$

For every set $A \subset N_{2}$ define a bijection $f_{A}: X \rightarrow X$ such that $f_{A}(L \backslash\{0\})=N_{1} \cup A$ and put $\tau_{A}:=\left\{f_{A}(U) \mid U \in \tau\right\}$. Clearly, $\tau_{A}$ is a topology on $X$ for every $A \subset N_{2}$. 
Moreover, for distinct sets $A, B \subset N_{2}$ the topologies $\tau_{A}$ and $\tau_{B}$ are distinct. Indeed, if $x \in A \backslash B$ then $\{x\}$ is open in $\left(X, \tau_{B}\right)$ because $x \notin f_{B}(L \backslash\{0\})$, but $\{x\}$ is not open in $\left(X, \tau_{A}\right)$ because $x \in f_{A}(L \backslash\{0\})$. Thus $\mathcal{F}_{0}^{\prime}=\left\{\tau_{A} \mid A \subset N_{2}\right\}$ is a subfamily of $\mathcal{F}_{0}$ with $\left|\mathcal{F}_{0}^{\prime}\right|=\left|\mathcal{P}\left(N_{2}\right)\right|=2^{\kappa}$ and hence we obtain $\left|\mathcal{F}_{0}\right| \geq 2^{\kappa}$.

In order to achieve $\left|\mathcal{F}_{0}^{*}\right| \geq \kappa^{+}$it suffices to determine $\kappa^{+}$non-homeomorphic compact Hausdorff spaces of size $\kappa$. Let $Z$ be the set of all ordinals strictly between the ordinals $\kappa$ and $\kappa^{+}$. Evidently, $|Z|=\kappa^{+}$. For each $\xi \in Z$ the ordinal space $\left[0, \omega^{\xi}\right]$ has size $\kappa$. (Note that $\omega^{\xi}$ is the ordinal power of the ordinal $\omega=\aleph_{0}$ with the ordinal exponent $\xi$.) With the help of Cantor derivatives and a little ordinal arithmetic it is not difficult to show that the spaces $\left[0, \omega^{\xi}\right]$ and $\left[0, \omega^{\xi^{\prime}}\right]$ are non-homeomorphic whenever $\xi, \xi^{\prime} \in Z$ are distinct. (Actually, the $\xi$-th derivative of $\left[0, \omega^{\xi}\right]$ is the singleton $\left\{\omega^{\xi}\right\}$ while the $\alpha$-th derivative of $\left[0, \omega^{\xi}\right]$ is infinite for every ordinal $\alpha<\xi$.)

Now let $X$ and $\mathcal{F}_{\omega}$ be as in Theorem 4 and assume $|X|=\kappa=\aleph_{0}$ for the present. Then $\mathcal{F}_{\omega}=\mathcal{F}_{0}$ and therefore $\left|\mathcal{F}_{\omega}^{*}\right|=\aleph_{1}$ because, by the well-known Mazurkiewicz-Sierpinski theorem [3], the ordinal spaces $\left[0, \omega^{\xi} \cdot n\right]$ form a complete system of representatives of the compact and countable Hausdorff spaces when $\xi$ runs through the countable ordinals and $n$ runs through the finite ordinals. Before we continue the proof of Theorem 4 we finish the proof of Theorem 2 by verifying its last statement. Let $\kappa>\aleph_{0}$. The Continuum Hypothesis $c=\aleph_{1}$ implies that $\kappa \geq c$ and therefore $\left|\mathcal{F}_{0}^{*}\right|=2^{\kappa}$ by Theorem 3. Consequently, if $\left|\mathcal{F}_{0}^{*}\right|=\kappa^{+}$could be proved then $2^{\kappa}=\kappa^{+}$could be proved by only assuming $2^{\aleph_{0}}=\aleph_{1}$ which is known to be impossible. On the other hand, if $\left|\mathcal{F}_{0}^{*}\right|>\kappa^{+}$could be proved then $2^{\kappa}>\kappa^{+}$would follow from $2^{\aleph_{0}}=\aleph_{1}$ which itself is a consequence of the consistent hypothesis that $2^{\lambda}=\lambda^{+}$holds for every transfinite cardinal $\lambda$.

Now we continue the proof of Theorem 4. Still assume that $|X|=\kappa>\aleph_{0}$. For each $\tau \in \mathcal{F}_{\omega}$ the space $(X, \tau)$ has weight $\aleph_{0}$ and hence it can be embedded in the Polish space $[0,1]^{\mathbb{N}}$. The compact subsets of the cube $[0,1]^{\mathbb{N}}$ are the closed ones and uncountable closed subsets of the cube $[0,1]^{\mathbb{N}}$ must have size $c$. Hence $\mathcal{F}_{\omega}=\emptyset$ when $\kappa \neq c$. It remains to settle the case $\kappa=c$. In this case we obtain $\left|\mathcal{F}_{\omega}\right| \leq c$ because on the one hand $\left|\mathcal{F}_{\omega}^{*}\right| \leq c$ since the cube $[0,1]^{\mathbb{N}}$ contains precisely $c$ compact subspaces, on the other hand there are at most $c$ homeomorphisms between second countable Hausdorff spaces. (Note that each continuous function from a Hausdorff space $S_{1}$ to a Hausdorff space $S_{2}$ is completely determined by its values on a dense subset of $S_{1}$.) Thus we are finished by producing $c$ mutually non-homeomorphic second countable compact Hausdorff spaces of size $c$. This is done by the following example.

Example 6. For each infinite set $M$ of positive integers define the space $Y_{M}$ as the topological sum of all Euclidean spaces $[0,1]^{n}$ where $n$ runs through $M$. Let $X_{M}=Y_{M} \cup\{\infty\}$ be the one point compactification of the locally compact Hausdorff space $Y_{M}$. Trivially, $\left|Y_{M}\right|=c$ for each of the $c$ sets $M$. If $M \neq M^{\prime}$ then $X_{M}$ and $X_{M^{\prime}}$ cannot be homeomorphic because, if $n \in M \backslash M^{\prime}$ then $[0,1]^{n}$ is a maximal connected subspace of $X_{M}$ which cannot be homeomorphic to anyone of the maximal connected subspaces of $X_{M^{\prime}}$ since these subspaces are precisely the spaces $[0,1]^{m}$ with $m \in M^{\prime}$ and the space $\{\infty\}$. Certainly, each space $X_{M}$ is second countable. 


\section{Proof of Theorem 3}

If $\kappa<c$ then $\mathcal{F}_{0} \cap \mathcal{F}_{4}=\emptyset$ because a connected and completely regular Hausdorff space must have size at least $c$ as already shown in the proof of Theorem 1 . Now let $\kappa \geq c$. Since $\left|\mathcal{F}_{0} \cap \mathcal{F}_{3} \cap \mathcal{F}_{4}\right| \leq\left|\mathcal{F}_{0}\right|=2^{\kappa}$ in view of Theorem 2 , in order to prove $\left|\left(\mathcal{F}_{0} \cap \mathcal{F}_{3} \cap \mathcal{F}_{4}\right)^{*}\right|=2^{\kappa}$ it is enough to find $2^{\kappa}$ mutually non-homeomorphic spaces of size $\kappa$ which are compact and connected and completely normal.

Let $\Omega_{1}$ be the well-ordered set of all countable ordinal numbers and $\bar{\Omega}_{1}=\Omega_{1} \cup\left\{\omega_{1}\right\}$ where $\omega_{1}=\max \bar{\Omega}_{1}$ is the first uncountable ordinal number. Evidently, $\left|\bar{\Omega}_{1}\right|=\aleph_{1}$. Let $\mathcal{L}$ be the (extended) long line constructed from $\bar{\Omega}_{1}$ by placing between each ordinal $\alpha \in \Omega_{1}$ and its successor $\alpha+1$ a copy of the open unit interval ]0,1[. In this way the set $\mathcal{L}$ becomes linearly ordered and $\mathcal{L}$ is a completely normal, compact and connected space with respect to the order topology. Define the dual space $\mathcal{L}^{*}$ of $\mathcal{L}$ in the following way. Let $g: \mathcal{L}^{*} \rightarrow \mathcal{L}$ be a bijection where $\mathcal{L}^{*}$ is a set disjoint from $\mathcal{L}$. Establish a linear ordering on $\mathcal{L}^{*}$ via $x<y$ for $x, y \in \mathcal{L}^{*}$ whenever $g(x)>g(y)$. One may regard $\mathcal{L}^{*}$ as the image of the ordered set $\mathcal{L}$ under a reflection in the point $0=\min \mathcal{L}$ or $\omega_{1}=\max \mathcal{L}$. The two spaces $\mathcal{L}$ and $\mathcal{L}^{*}$ are trivially homeomorphic ( $g$ is a homeomorphism), but the two ordered sets $\mathcal{L}$ and $\mathcal{L}^{*}$ are not isomorphic. Moreover, writing $A \cong B$ when the ordered sets $A$ and $B$ are isomorphic,

$$
\{x \in \mathcal{L} \mid x \leq y\} \supsetneqq\left\{x \in \mathcal{L}^{*} \mid x \leq z\right\} \quad \text { if } z \in \mathcal{L}^{*} \text { and } 0 \neq y \in \mathcal{L} .
$$

Actually, if $\omega_{1} \neq u \in \mathcal{L}$ then there exists a strictly increasing function from $\Omega_{1}$ to $\{x \in$ $\mathcal{L} \mid x \geq u\}$. Equivalently, if $z \in \mathcal{L}^{*}$ and $z \neq \min \mathcal{L}^{*}$ then there exists a strictly decreasing function from $\Omega_{1}$ to $\left\{x \in \mathcal{L}^{*} \mid x \leq z\right\}$. There cannot exist a strictly decreasing function from $\Omega_{1}$ to the long line $\mathcal{L}$ since $|A| \leq \aleph_{0}$ for every strictly monotone function from any $A \subset \Omega_{1}$ to the Euclidean interval ]0, 1[ and, naturally, there does not exist an infinite chain $\alpha_{1}>\alpha_{2}>\alpha_{3}>\ldots$ of ordinal numbers. Therefore (4.1) must be true. It should also be noted that

$$
\{x \in \mathcal{L} \mid x \leq y\} \supsetneqq \mathcal{L} \quad \text { if } \omega_{1} \neq y \in \mathcal{L} .
$$

Indeed, for $y \in \mathcal{L}$ there exists a strictly increasing function from $\Omega_{1}$ to $\{x \in \mathcal{L} \mid x \leq y\}$ if and only if $y=\omega_{1}$. (Note that $\left\{\alpha \in \bar{\Omega}_{1} \mid \alpha<\beta\right\}$ is countable for all $\beta \in \Omega_{1}$.)

Now regard $\kappa$ as an initial ordinal and let $\Omega$ be the (well-ordered) set of all ordinal numbers $\leq \kappa$, whence $|\Omega|=\kappa$. Let $\mathcal{J}=\mathcal{L} \backslash\{\min \mathcal{L}, \max \mathcal{L}\}$ and $\mathcal{J}^{*}=\mathcal{L}^{*} \backslash\left\{\min \mathcal{L}^{*}, \max \mathcal{L}^{*}\right\}$. Let $\Sigma$ be the family of all functions from $\Omega \backslash\{0, \kappa\}$ to the set $\{0,1\}$. Trivially, $|\Sigma|=2^{\kappa}$. Thus it suffices to construct for each $\sigma \in \Sigma$ a compact, connected and completely normal space $X_{\sigma}$ of size $\kappa$ such that the spaces $X_{\sigma}$ and $X_{\sigma^{\prime}}$ are non-homeomorphic whenever $\sigma, \sigma^{\prime} \in \Sigma$ are distinct. In order to achieve this, for each $\sigma \in \Sigma$ we create a sort of hyper-long line $X_{\sigma}$ constructed from $\Omega$ by placing between each ordinal $\alpha \in \Omega \backslash\{\kappa\}$ and its successor $\alpha+1$ a copy of either the ordered set $\mathcal{J}$ or the ordered set $\mathcal{J}^{*}$ in the following way. Between the ordinal 0 and the ordinal 1 we place $\mathcal{J}$. For $\alpha \in \Omega \backslash\{0, \kappa\}$, between $\alpha$ and $\alpha+1$ we place a copy of $\mathcal{J}$ when $\sigma(\alpha)=0$ and a copy of $\mathcal{J}^{*}$ when $\sigma(\alpha)=1$. It is evident that in this way the ordering on $X_{\sigma}$ is complete and that there is no pair of consecutive points. So $X_{\sigma}$ becomes a compact, connected, completely normal space of size $\kappa$. For each $\sigma \in \Sigma$ we have $\min X_{\sigma}=0$ and $\max X_{\sigma}=\kappa$. In order to avoid ambiguity, we write $[a, b]_{\sigma}$ for $\left\{x \in X_{\sigma} \mid a \leq x \leq b\right\}$ when $a, b \in X_{\sigma}$ and $a \leq b$. 
Now choose distinct sequences $\sigma, \sigma^{\prime} \in \Sigma$. In order to show that $X_{\sigma}$ and $X_{\sigma^{\prime}}$ are not homeomorphic, suppose that there is a homeomorphism $f: X_{\sigma} \rightarrow X_{\sigma^{\prime}}$. First we show that $f$ must be an order isomorphism. Indeed, $f$ maps compact sets onto compact sets and connected sets onto connected sets. The connected sets in the ordered space $X_{\sigma}$ and $X_{\sigma^{\prime}}$, respectively, are precisely the intervals, the compact and connected sets are precisely the closed intervals. Since any compact and connected set $[u, v]$ remains connected after removing the two distinct points $u$ and $v$, we obtain $f\left([x, y]_{\sigma}\right)=[a, b]_{\sigma^{\prime}}$ with $\{a, b\}=$ $\{f(x), f(y)\}$ whenever $x, y \in X_{\sigma}$ and $x<y$. Moreover, since $f\left([0, x]_{\sigma}\right) \subset f\left([0, y]_{\sigma}\right)$ whenever $x \leq y$, the function $f$ must be either increasing or decreasing. If $f$ is decreasing then $f(0)=\kappa$. But this is impossible. Indeed, in the space $X_{\sigma^{\prime}}$ each open neighborhood of the point $\kappa$ contains a copy of $\mathcal{L}$ or $\mathcal{L}^{*}$. On the other side (literally), in the space $X_{\sigma}$ the point 0 has an open neighborhood homeomorphic with the Euclidean space [0, 1[ which, of course, cannot contain a copy of $\mathcal{L}$ or $\mathcal{L}^{*}$. Thus $f(0)=0$ and $f$ is increasing.

Now let $\eta$ be the smallest ordinal in $\Omega \backslash\{0, \kappa\}$ such that $\sigma(\eta) \neq \sigma^{\prime}(\eta)$ and, say, $\sigma(\xi)=0$ when $0<\xi \leq \eta$. Then the intervals $[0, \eta]_{\sigma},[0, \eta]_{\sigma^{\prime}}$ and their orderings coincide. First we observe that we must have $f(\eta) \leq \eta$. Indeed, $f(\eta)>\eta$ implies that $[\eta, f(\eta)]_{\sigma^{\prime}}$ contains a copy $C$ of $\left\{x \in \mathcal{L}^{*} \mid x \leq z\right\}$ with $z \neq \min \mathcal{L}^{*}$ and $\eta=\min C$ and therefore we can find a strictly decreasing function from $\Omega_{1}$ to $[\eta, f(\eta)]_{\sigma^{\prime}}$. But we cannot find such a function from $\Omega_{1}$ to $f^{-1}\left([\eta, f(\eta)]_{\sigma^{\prime}}\right)$ since $f^{-1}\left([\eta, f(\eta)]_{\sigma^{\prime}}\right) \subset[0, \eta]_{\sigma}$ and $[0, \eta]_{\sigma}$ consists only of ordinal numbers with copies of $\mathcal{J}$ between them.

Secondly, we prove by induction that $f(\xi)=\xi$ for each ordinal $\xi \leq \eta$, whence $f(\eta)=\eta$. Suppose that $f(\alpha)=\alpha$ is already verified for an ordinal $\alpha<\eta$. Then the interval $[0, \alpha+1]_{\sigma}$ is the union of $[0, \alpha]_{\sigma}$ and one copy $\mathcal{L}^{\prime}$ of $\mathcal{L}$ where the maximum $\alpha$ of $[0, \alpha]_{\sigma}$ is identified with the minimum of $\mathcal{L}^{\prime}$ and $x<y$ whenever $x \in[0, \alpha]_{\sigma}$ and $\alpha \neq y \in \mathcal{L}^{\prime}$. Since $f(\alpha)=\alpha$ and $f(\eta) \leq \eta, f$ is an increasing function on the domain $\mathcal{L}^{\prime}$ with $f\left(\mathcal{L}^{\prime}\right) \subset$ $[\alpha, \eta]_{\sigma^{\prime}}$ and $f\left(\min \mathcal{L}^{\prime}\right)=\alpha$. In order to arrive at $f(\alpha+1)=\alpha+1$ as requested, we have to verify $f\left(\max \mathcal{L}^{\prime}\right)=\alpha+1$. Well, $f\left(\max \mathcal{L}^{\prime}\right)<\alpha+1$ implies $\mathcal{L} \cong f\left(\mathcal{L}^{\prime}\right) \cong\{x \in \mathcal{L} \mid x \leq$ $y$ \} for some $y \in \mathcal{L} \backslash\left\{\omega_{1}\right\}$ contrarily to (4.2). On the other hand, $f\left(\max \mathcal{L}^{\prime}\right)>\alpha+1$ implies $\beta=f^{-1}(\alpha+1)<\alpha+1$ and hence $[\alpha, \beta]_{\sigma} \cong f\left([\alpha, \beta]_{\sigma}\right)=[\alpha, \alpha+1]_{\sigma^{\prime}} \cong \mathcal{L}$ contrarily to (4.2) as well. If $\lambda \leq \eta$ is a limit ordinal such that $f(\alpha)=\alpha$ is already proved for all ordinals $\alpha<\lambda$ then $f(\lambda)=\lambda$ since $f(0)=0$ and $[0, \lambda]_{\sigma} \backslash\{\lambda\}=\bigcup\left\{[0, \alpha]_{\sigma} \mid \alpha \in \Omega \wedge \alpha<\lambda\right\}$. Having realized that $f(\eta)=\eta$ and hence $f\left([0, \eta]_{\sigma}\right)=[0, \eta]_{\sigma^{\prime}}$, we arrive at the desired contradiction to the assumption $X_{\sigma} \cong X_{\sigma^{\prime}}$ for $\sigma \neq \sigma^{\prime}$. Actually, since $\sigma(\eta)=0$ and $\sigma^{\prime}(\eta)=1$, the interval $[\eta, \eta+1]_{\sigma}$ is a copy of $\mathcal{L}$ while the interval $[\eta, \eta+1]_{\sigma^{\prime}}$ is a copy of $\mathcal{L}^{*}$ and hence both $f(\eta+1) \leq \eta+1$ and $f(\eta+1)>\eta+1$ are repugnant with (4.1).

\section{References}

[1] Comfort, W.W.; Negrepontis, S.: The Theory of Ultrafilters. Springer 1974.

[2] Juhász, I.: Cardinal Functions in Topology. Mathematisch Centrum Amsterdam 1983.

[3] Mazurkiewicz, S.; Sierpinski, W.: Contribution à la topologie des ensembles dénombrables. Fund. Math. 1 (1920), 17-27.

[4] Steen, L.A.; Seebach Jr., J.A.: Counterexamples in Topology. Dover 1995.

Gerald Kuba, Institut für Mathematik, Universität für Bodenkultur A-1180 Wien, Österreich; e-mail: gerald.kuba@boku.ac.at 